\title{
Follow-Up of Bone Mineral Density Changes in de novo Kidney Transplant Recipients Treated with Two Doses of the Receptor Activator of Nuclear Factor KB Ligand Inhibitor Denosumab
}

\author{
Claudia Kobel $^{a} \quad$ Diana Frey ${ }^{b} \quad$ Nicole Graf ${ }^{c} \quad$ Rudolf P. Wüthrich ${ }^{a}$ \\ Marco Bonani ${ }^{a}$ \\ ${ }^{a}$ Division of Nephrology, University Hospital, Zürich, Switzerland;

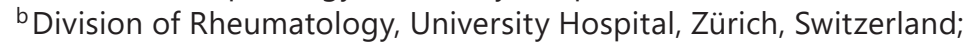 \\ ${ }^{\mathrm{C}}$ Graf Biostatistics, Winterthur, Switzerland
}

\section{Keywords}

Bone mineral density $\cdot$ Denosumab $\cdot$ Kidney transplantation $\cdot$ Osteoporosis

\begin{abstract}
Background: Studies in women with post-menopausal osteoporosis have shown that discontinuation of treatment with denosumab leads to an increased risk of vertebral fractures because of rebound bone turnover and rapid loss of bone mineral density (BMD). Methods: In a post hoc analysis of the Prolia for Osteoporosis of Transplant Operated Patient study, we analyzed the effect of denosumab withdrawal on BMD changes. Twenty-five de novo kidney transplant recipients (KTR) who were treated for 1 year with 2 six-monthly doses of denosumab on top of standard treatment (daily calcium and vitamin $D$ ) were compared to a control group of 29 KTR who received standard treatment alone. BMD changes were analyzed by repeated dual-energy X-ray absorptiometry shortly after transplantation (baseline), after 6 and 12 months (active treatment phase) and after 2-6.5 years (follow-up phase). Results: The average BMD at the lumbar spine declined markedly after discontinuation of treatment with denosumab but increased again thereafter. Thus, the average monthly change in lumbar spine BMD from month 12 onward was only $0.1 \pm 2.8 \%$ o in the denosumab group but $1.5 \pm 1.9 \%$ o in the control group $(p=0.021)$. The average monthly change in lumbar spine BMD from baseline to follow-up was similar in the control and denosumab group $(1.1 \pm 1.2 \%$ o vs. $1.5 \pm 2.4 \%$, $\mathrm{p}=0.788)$. Similar results were seen at the total hip. Conclusions: In de novo KTR treated with
\end{abstract}




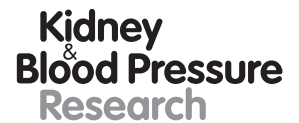

Research

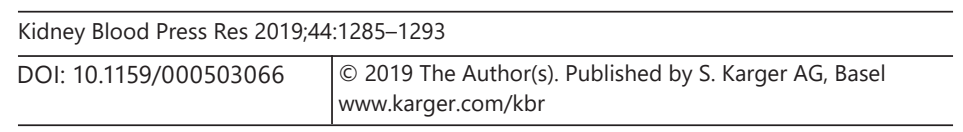

Kobel et al.: Denosumab after Renal Transplantation

2 doses of denosumab, we detect a marked decrease in lumbar spine and hip BMD when denosumab is discontinued. Denosumab treatment should therefore not be discontinued without considering an alternative antiresorptive treatment.

(C) 2019 The Author(s)

Published by S. Karger AG, Basel

\section{Introduction}

Loss of bone mineral density (BMD) is common in kidney transplant recipients (KTR), in particular in the first year after transplantation, due to the effects of immunosuppressive medication (especially glucocorticoids), persistence of elevated PTH and FGF-23 levels, and low vitamin D status [1]. Hence, there is also a heightened risk for developing or worsening osteoporosis and bone fractures [2,3]. Supplementation with calcium and vitamin D and its analogues as well as treatment with bisphosphonates are therapeutic options to improve the loss of bone mass after renal transplantation.

Denosumab is a fully human monoclonal antibody with high affinity and specificity for Receptor Activator of Nuclear Factor $\kappa B$ ligand (RANKL), which inhibits osteoclast formation, function and survival. Denosumab thereby reduces bone resorption and improves BMD, and is therefore used for treatment of osteoporosis and prevention of fractures [4-7]. The pivotal randomized, double-blind, placebo-controlled, phase 3 Fracture Reduction Evaluation of Denosumab in Osteoporosis Every 6 Months trial in women with postmenopausal osteoporosis showed that treatment with denosumab led to a markedly reduced incidence in new vertebral fractures, nonvertebral fractures and hip fractures when compared to placebo [8]. In KTR, we have recently shown that denosumab ( $60 \mathrm{mg}$ subcutaneously, given 2 weeks after surgery and after 6 months) effectively increased BMD in the first year after kidney transplantation [9].

Several studies in women with post-menopausal osteoporosis, including a post hoc analysis of the Fracture Reduction Evaluation of Denosumab in Osteoporosis Every 6 Months trial and its extensions [10-12], have shown that discontinuation of treatment with denosumab leads to an increased risk of vertebral fractures due to enhanced bone turnover and rapid loss of BMD. After 7 or 10 years of treatment with denosumab, the discontinuation of the treatment resulted in a rapid decrease in BMD over 1 year [10]. Miller et al. [7] showed that the inhibitory effects of denosumab on bone turnover were reversed after discontinuation of denosumab but restored with subsequent retreatment. These findings are in line with several case reports of patients who developed multiple new vertebral fractures after discontinuation of denosumab [13-17] as well as a significant bone loss [18] after the withdrawal of denosumab.

The aim of the present study was to extend these findings to the setting of renal transplantation. Thus, we analyzed the follow-up changes in BMD in KTR who received $60 \mathrm{mg}$ denosumab shortly after and 6 months after transplantation to examine whether there is a significant loss in BMD upon denosumab withdrawal in these patients.

\section{Materials and Methods}

Details of the Prolia for Osteoporosis of Transplant Operated Patients (POSTOP) study have been reported previously [9]. In brief, we recruited a total of 90 KTR that had been transplanted less than 4 weeks ago (mean 16 days) to examine 0.5 and 1-year changes in BMD at the lumbar spine and the hip by dual-energy X-ray absorptiometry (DXA). The patients were randomly assigned (1:1) to open-label treatment with denosumab $60 \mathrm{mg}$ subcutaneously at baseline and after 6 months $(n=46)$ or no treatment $(n=44)$. Treatment with denosumab 
Fig. 1. Scheme depicting the time intervals for calculation of monthly change in BMD.

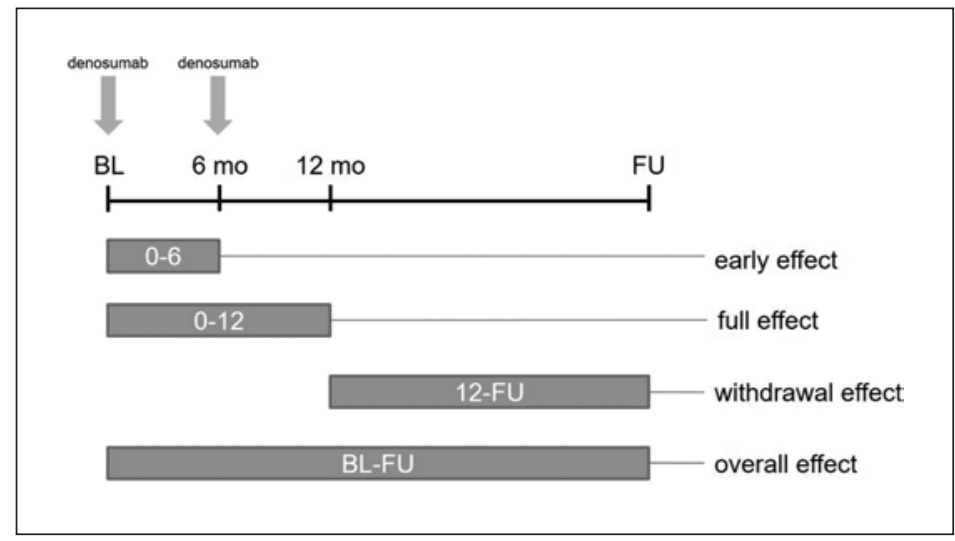

was terminated after 1 year. All patients were prescribed daily calcium (1,000 mg) and vitamin D (800 IU). All participants gave their written informed consent. The study was designed and conducted to conform to the principles of the Declaration of Helsinki, was approved by the local Ethics Committee (IRB approval number 2011-0032), and was registered on ClinicalTrials.gov, number NCT01377467.

In the present retrospective analysis of the POSTOP study, we analyzed the course of BMD in a subset of patients that had at least one follow-up DXA. Thus, 25 de novo KTR with denosumab withdrawal could be compared to 29 control KTR who had not received denosumab. After the initial 1-year study period, patients were seen at our transplant center or by their local nephrologists for regular clinical and laboratory transplant follow-up. The decision to perform additional DXA was made by the treating nephrologist and took place between 3 and 7.5 years after baseline.

To quantify the changes of BMD over time we calculated the average monthly change in lumbar spine and hip BMD for 4 different time intervals: (1) from baseline to 6 months after baseline to calculate the early response to denosumab; (2) from baseline to 12 months after baseline to calculate the full response to ongoing denosumab treatment; (3) from 12 months onward to follow-up visit to estimate the withdrawal effect and (4) from baseline to followup visit to quantify the overall effect of a 1-year "prophylactic" denosumab treatment regime (Fig. 1).

\section{Statistics}

Baseline characteristics were compared between the treatment groups with a Wilcoxon rank sum test for continuous data, and a chi-square or Fisher's exact test for categorical data. The percentage change of BMD at the lumbar spine and the hip was divided by months after baseline visit or after 12 month's visit, and was compared between the treatment groups with a Wilcoxon rank sum test. All analyses were performed in the R programming language (version 3.3.3) [19]. The package "ggplot2" [20] was used to plot the figures and fit the total lumbar and hip BMD by time, using local fitting (loess) with a span (degree of smoothing) of 0.85 .

\section{Results}

From the initial 90 KTR in the POSTOP study we were able to obtain follow-up DXA measurements in 54 patients. A total of 45 KTR had one, and 9 patients had 2 additional DXAs in the follow-up phase; 25 patients had been treated with denosumab for 1 year and 29 patients had not received denosumab. 
Table 1. Demographic and baseline characteristics of transplanted patients

\begin{tabular}{lcc}
\hline Characteristics & Control group $(n=29)$ & Denosumab group $(n=25)$ \\
\hline Age, years & $49.8 \pm 13.1$ & $51.4 \pm 13.0$ \\
Gender, male, $n$ (\%) & $13(45)$ & $18(72)$ \\
BMI, kg/m² & $24.7 \pm 4.5$ & $25.7 \pm 4.7$ \\
Pre-transplant dialysis mode, $n$ (\%) & & \\
$\quad$ Hemodialysis & $22(76)$ & $13(52)$ \\
$\quad$ Peritoneal dialysis & $6(21)$ & $4(16)$ \\
$\quad$ Pre-emptive transplantation & $1(3)$ & $8(32)$ \\
Deceased donor transplantation, $n(\%)$ & $18(62)$ & $9(36)$ \\
Repeat transplantation, $n$ (\%) & $5(17)$ & $4(16)$ \\
eGFR, mL/min/1.73 m ${ }^{2}$ & $56.1 \pm 16.7$ & $54.5 \pm 15.6$ \\
PTH, ng/L & $126.7 \pm 87.4$ & $182.1 \pm 196.1$ \\
25-hydroxyvitamin D, $\mu \mathrm{g} / \mathrm{L}$ & $19.0 \pm 9.2$ & $16.4 \pm 9.2$ \\
Total lumbar spine BMD, g/cm ${ }^{2}$ & $0.917 \pm 0.135$ & $0.994 \pm 0.138$ \\
Total hip BMD, g/cm ${ }^{2}$ & $0.843 \pm 0.093$ & $0.903 \pm 0.131$ \\
Osteopenic, $n$ (\%) & $16(57)$ & $8(32)$ \\
Osteoporotic, $n$ (\%) & $6(21)$ & $3(12)$ \\
\hline
\end{tabular}

Values show means \pm SD if not otherwise stated.

eGFR, estimated glomerular filtration rate; BMD, bone mineral density; BMI, body mass index.

The baseline patient characteristics of the 54 KTR are shown in Table 1 . The study population had a mean age of $51 \pm 13$ years; $57.4 \%$ were male and all were of white ethnicity; mean baseline eGFR was $55.4 \pm 16.0 \mathrm{~mL} / \mathrm{min} / 1.73 \mathrm{~m}^{2}$. All patients were initially treated with triple immunosuppression, including a calcineurin antagonist, mycophenolate mofetil and glucocorticosteroids. Of all patients, 24 (44\%) had osteopenia and another 9 (17\%) had osteoporosis. The baseline characteristics of the subset of the POSTOP study patients $(n=54)$ did not differ from the original study population ( $n=90$; data not shown).

The 2 study groups were generally well balanced except that more patients in the control group were osteopenic (57\%) or osteoporotic (21\%) than in the denosumab group (32 and $12 \%$, respectively). Also, there were less men $(n=13,45 \%)$ in the control group than in the denosumab group $(n=18,72 \%)$ and there were less living donor transplantations in the control group (38\%) than in the denosumab group (64\%). A total of 22 patients $(76 \%)$ in the control group were on hemodialysis and $1(3 \%)$ was a pre-emptive transplantation, while 13 $(52 \%)$ in the denosumab group were on hemodialysis and $8(32 \%)$ had a pre-emptive transplantation. The biochemical parameters of bone metabolism at baseline were balanced between the 2 treatment groups. In particular, patients had similar levels of PTH and 25-hydroxyvitamin D.

Figure 2 depicts the change of total lumbar BMD over time by the randomization group. Locally weighted regression (loess) analysis revealed that the previously increased BMD at the lumbar spine (baseline to 12 months) declined markedly after discontinuation of the treatment with denosumab, suggesting that the treatment effect was lost within 6-12 months. The BMD increased again thereafter, but at a lower rate.

Figure 3 depicts the change of total hip BMD over time by randomization group. A similar decrease of BMD was seen after discontinuation of denosumab treatment, with a slower increase of BMD thereafter. This demonstrates that the rapid decrease of BMD after denosumab withdrawal is occurring at different skeletal sites.

In Figure 4, it is shown that denosumab very effectively increased BMD at the lumbar spine and total hip in the first 6 and 12 months. When averaging BMD from baseline to the 
Kidney

Blood Pressure

Research

Fig. 2. Change of total lumbar BMD $\left(\mathrm{g} / \mathrm{cm}^{2}\right)$ over time by the randomization group. Thin lines show the course of total lumbar BMD of individual patients of the original POSTOP group $(n=88)$; the thick black line shows the average trend by using locally weighted regression analysis (loess); the dashed line indicates 12 months after baseline visit (end of denosumab effect). BMD, bone mineral density.

Fig. 3. Change of total hip BMD $\left(\mathrm{g} / \mathrm{cm}^{2}\right)$ over time by the randomization group. Thin lines show course of total hip BMD of individual patients of the original POSTOP group ( $n=88$ ); the thick black line shows the average trend by using locally weighted regression analysis (loess); the dashed line indicates 12 months after baseline visit (end of denosumab effect). BMD, bone mineral density.

Fig. 4. Monthly changes in \%o from baseline to 6 months (Comparison 1); from baseline to 12 months (Comparison 2); from month 12 to follow-up visit (Comparison 3); and from baseline to follow-up visit (Comparison 4). Bar graphs show the mean and SEM. BMD, bone mineral density.

\begin{tabular}{l|l}
\hline \multicolumn{2}{l}{ Kidney Blood Press Res 2019;44:1285-1293 } \\
\hline DOI: 10.1159/000503066 & $\begin{array}{l}\text { (c) 2019 The Author(s). Published by S. Karger AG, Basel } \\
\text { www.karger.com/kbr }\end{array}$ \\
\hline
\end{tabular}
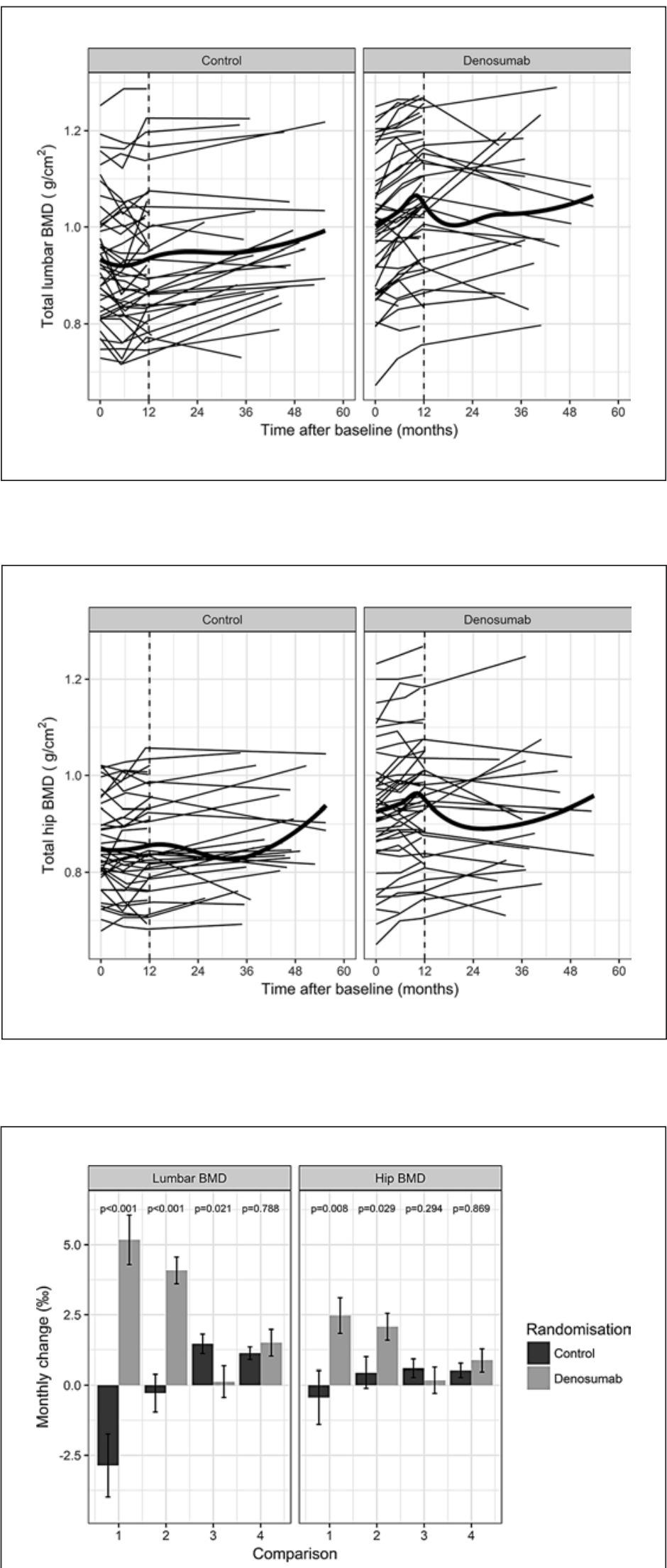


\section{Kidney \\ Blood Pressure \\ Research}

Table 2. Subanalysis of monthly lumbar spine BMD change (in \%o) 12 months until follow-up

\begin{tabular}{l|l}
\hline Kidney Blood Press Res 2019;44:1285-1293 \\
\hline DOI: 10.1159/000503066 & $\begin{array}{l}\text { @ 2019 The Author(s). Published by S. Karger AG, Basel } \\
\text { www.karger.com/kbr }\end{array}$ \\
\hline
\end{tabular}

\begin{tabular}{lcc}
\hline & Control $(n=29)$ & Denosumab $(n=25)$ \\
\hline Gain group & $8(28)$ & $5(16)$ \\
Loss group & $0(0)$ & $3(12)$ \\
Stable group & $21(72)$ & $18(72)$ \\
\hline
\end{tabular}

Number of patients and \% of all patients in each group are indicated. BMD, bone mineral density.

follow-up visit (median 41.8 months, IQR 13.6 months), the change of total lumbar BMD was not significantly different in the control and denosumab groups $(1.1 \pm 1.2 \%$ vs. $1.5 \pm 2.4 \%$, $p=0.788$ ). Likewise, the total hip BMD average monthly change was similar in both groups $(0.5 \pm 1.3 \%$ vs. $0.9 \pm 2.0 \%$ o, $p=0.869)$. Of importance, the average monthly change in lumbar spine BMD from month 12 onward was $1.5 \pm 1.9 \%$ in the control group, but substantially less at $0.1 \pm 2.8 \%$ in the denosumab group ( $p=0.021)$. Likewise, the average monthly change in hip BMD tended to be higher in the control than that in the denosumab group, albeit not significantly ( $0.6 \pm 1.7 \%$ vs. $0.2 \pm 2.3 \%$ o $p=0.294)$.

To consolidate these data, we performed a subanalysis by dividing the study patients in both groups according to 3 different lumbar spine BMD change categories (from month 12 to follow-up) as follows: (1) patients with $>+2.5 \%$ per month BMD change (gain group); (2) patients with -2.5 to $+2.5 \%$ o per month BMD change (stable group); and 3) patients with less than $-2.5 \%$ per month BMD change (loss group). As can be seen in Table 2, denosumab treatment compared with control resulted in more patients in the loss group (12 vs. $0 \%$ ) and fewer in the gain group (16 vs. $28 \%$ ), confirming our main data.

We were also interested to determine whether the use of steroids was similar in both treatment groups. Previously we reported that during the 12-month treatment phase the cumulative amount of steroids was similar ( $3.760 \mathrm{vs}$. $3.974 \mathrm{~g}$ in control vs. denosumab group). We calculated the cumulative amount of steroids from 12 months until follow-up and did not find a significant difference (control group $0.727 \pm 1.722 \mathrm{~g}$, denosumab group $1.575 \pm$ $2.779 \mathrm{~g}, p=0.293$ ).

\section{Discussion}

Densoumab is a first-in-class anti-osteoporotic drug, which is highly effective in preventing vertebral and non-vertebral fractures in postmenopausal women with osteoporosis [8]. When compared with bisphosphonates in this patient population, it has superior efficacy on BMD at all measured skeletal sites [21], although a recently published meta-analysis could not show a significantly reduced fracture risk under denosumab compared to bisphosphonates despite higher gains in BMD; thus, there has not been a documented superiority on fracture risk reduction so far $[22,23]$. Recently, several studies in women with post-menopausal osteoporosis $[10,12,24]$ have shown that discontinuation of treatment with denosumab leads to enhanced bone turnover and rapid loss of BMD within a relatively short time with a substantially increased incidence of vertebral fractures.

In this post hoc analysis of the POSTOP study, we also detected a drop in lumbar and hip BMD in de novo KTR when denosumab is discontinued, most likely because of upregulation of osteoclastogenesis and osteoclast activity [25]. Thus, the gain in BMD in the 12 months of treatment was lost upon discontinuation of denosumab. Fortunately however, at an average follow-up of 42 months the BMD was not inferior to the control group. We did not see a signif- 


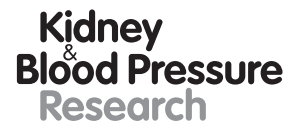

Kidney
Blood Pressure
Research \begin{tabular}{l|l}
\hline Kidney Blood Press Res 2019;44:1285-1293 \\
\hline DOI: 10.1159/000503066 & $\begin{array}{l}\text { @ 2019 The Author(s). Published by S. Karger AG, Basel } \\
\text { www.karger.com/kbr }\end{array}$ \\
\hline
\end{tabular}

Kobel et al.: Denosumab after Renal Transplantation

icant difference in the cumulative amount of steroids that the patients received in the 2 treatment groups, excluding that steroids might have affected the results. As there were less number of women and less recipients of a deceased donor kidney transplant in the denosumab group, we may have underestimated the magnitude of the denosumab withdrawal effect.

It has been suggested that treatment with bisphosphonates might antagonize the enhanced bone turnover after withdrawal of denosumab due to their high affinity for bone hydroxyapatite and long half-life within the skeleton, persisting for months or even years [26]. Indeed, several small studies in postmenopausal women showed that switching to bisphosphonates, after having been treated with denosumab, was associated with lower or only slightly elevated bone turnover biomarkers such as C-telopeptides or N-telopeptide, a stabilized BMD after having gained BMD under denosumab and thus less bone loss [27-29].

Currently, it is therefore advised to administer bisphosphonates after discontinuation of denosumab to prevent rapid bone loss in postmenopausal women. Bisphosphonates can be given orally like alendronate, which has been shown to maintain BMD after stopping denosumab [30] or intravenously like zoledronate or risedronate [31]. Very recently it was shown that de novo KTR with a high fracture risk, who received zoledronic acid in the first year after kidney transplantation, had a significant positive effect of zoledronate with an increase of BMD in both lumbar spine and total hip sites without the risk of inducing adynamic bone disease [32]. Due to the marked but reversible inhibition of bone remodeling under denosumab therapy and due to the higher possibility of impaired renal function in the first year after transplantation, starting therapy with denosumab in de novo KTR followed by bisphosphonate treatment might therefore be an excellent choice to prevent the loss of BMD after kidney transplantation.

Admittedly, our study has several limitations. The sample size was relatively small and there were sizable baseline differences between the groups, which could influence BMD. In addition loss to follow-up presents a possible selections bias due to considering patients with follow-up DXA scans as higher risk patients by their local nephrologists. Furthermore, the follow-up DXA measurements were not performed in a systematic fashion at defined time intervals, and this resulted in different follow-up periods, thus this analysis is limited in determining the rate of bone loss following the withdrawal of denosumab. To minimize this limitation, we extended the loess analysis by expressing BMD changes on a monthly basis at defined time intervals, and this confirmed the initial findings.

Another important limitation of our study resides in the fact that many KTR had only osteopenia or relatively normal BMD. We had initially expected that many more patients would develop osteoporosis within 1 year after transplantation, but this was not the case, possibly because of ample calcium and vitamin D substitution. Continuing denosumab beyond 1 year would not make sense in these patients with preserved BMD. Based on our data we would recommend that only those KTR with osteoporosis be treated with denosumab, and that in these patients continuous treatment would be required beyond 1 year to avoid a rapid decline in BMD after the cessation of treatment.

In conclusion, de novo KTR treated with 2 doses of denosumab in the first year show a marked decrease in lumbar spine and hip BMD when denosumab is discontinued. Due to the retrospective study design, these findings are suggestive and consistent with the literature, but further studies need to be performed. Whether subsequent bisphosphonate treatment might be effective to prevent the probable decline in BMD after denosumab discontinuation would need to be tested in a prospective clinical study. 


\section{Acknowledgments}

We thank the study patients for their participation.

\section{Disclosure Statement}

Dr. Nicole Graf reports personal fees from Astellas Pharma AG, outside the submitted work. The other authors have no conflicts of interest to disclose.

\section{Funding Sources}

This work was supported by the University Hospital Zürich and the University Zürich. There was no commercial funding for this study.

\section{Author Contributions}

M.B. and R.P.W. designed the study, analyzed the data, and assisted in the preparation of the manuscript. C.K. contributed to data analysis and wrote the initial draft of the manuscript. D.F. contributed to data analysis and interpretation and assisted in the preparation of the manuscript. N.G. performed the statistical analysis and assisted in the preparation of the manuscript.

\section{References}

1 Cunningham J. Posttransplantation bone disease. Transplantation. 2005 Mar;79(6):629-34.

2 Vautour LM, Melton LJ 3rd, Clarke BL, Achenbach SJ, Oberg AL, McCarthy JT. Long-term fracture risk following renal transplantation: a population-based study. Osteoporos Int. 2004 Feb;15(2):160-7.

3 Nikkel LE, Hollenbeak CS, Fox EJ, Uemura T, Ghahramani N. Risk of fractures after renal transplantation in the United States. Transplantation. 2009 Jun;87(12):1846-51.

4 Bone HG, Bolognese MA, Yuen CK, Kendler DL, Wang H, Liu Y, et al. Effects of denosumab on bone mineral density and bone turnover in postmenopausal women. J Clin Endocrinol Metab. 2008 Jun;93(6):2149-57.

5 Lewiecki EM, Miller PD, McClung MR, Cohen SB, Bolognese MA, Liu Y, et al.; AMG 162 Bone Loss Study Group. Two-year treatment with denosumab (AMG 162) in a randomized phase 2 study of postmenopausal women with low BMD. J Bone Miner Res. 2007 Dec;22(12):1832-41.

6 McClung MR, Lewiecki EM, Cohen SB, Bolognese MA, Woodson GC, Moffett AH, et al.; AMG 162 Bone Loss Study Group. Denosumab in postmenopausal women with low bone mineral density. N Engl J Med. 2006 Feb; 354(8): 821-31.

7 Miller PD, Bolognese MA, Lewiecki EM, McClung MR, Ding B, Austin M, et al.; Amg Bone Loss Study Group. Effect of denosumab on bone density and turnover in postmenopausal women with low bone mass after longterm continued, discontinued, and restarting of therapy: a randomized blinded phase 2 clinical trial. Bone. 2008 Aug;43(2):222-9.

8 Cummings SR, San Martin J, McClung MR, Siris ES, Eastell R, Reid IR, et al.; FREEDOM Trial. Denosumab for prevention of fractures in postmenopausal women with osteoporosis. N Engl J Med. 2009 Aug;361(8):756-65.

9 Bonani M, Frey D, Brockmann J, Fehr T, Mueller TF, Saleh L, et al. Effect of twice-yearly denosumab on prevention of bone mineral density loss in de novo kidney transplant recipients: a randomized controlled trial. Am J Transplant. 2016 Jun;16(6):1882-91.

10 Popp AW, Varathan N, Buffat H, Senn C, Perrelet R, Lippuner K. Bone mineral density changes after 1 year of denosumab discontinuation in postmenopausal women with long-term denosumab treatment for osteoporosis. Calcif Tissue Int. 2018 Jul;103(1):50-4.

11 Cummings SR, Ferrari S, Eastell R, Gilchrist N, Jensen JB, McClung M, et al. Vertebral fractures after discontinuation of denosumab: a post hoc analysis of the randomized placebo-controlled FREEDOM trial and its extension. J Bone Miner Res. 2018 Feb;33(2):190-8. 
12 Zanchetta MB, Boailchuk J, Massari F, Silveira F, Bogado C, Zanchetta JR. Significant bone loss after stopping long-term denosumab treatment: a post FREEDOM study. Osteoporos Int. 2018 Jan;29(1):41-7.

13 Lamy O, Gonzalez-Rodriguez E, Stoll D, Hans D, Aubry-Rozier B. Severe rebound-associated vertebral fractures after denosumab discontinuation: 9 clinical cases report. J Clin Endocrinol Metab. 2017 Feb;102(2):354-8.

14 Anastasilakis AD, Makras P. Multiple clinical vertebral fractures following denosumab discontinuation. Osteoporos Int. 2016 May;27(5):1929-30.

15 Popp AW, Zysset PK, Lippuner K. Rebound-associated vertebral fractures after discontinuation of denosumabfrom clinic and biomechanics. Osteoporos Int. 2016 May;27(5):1917-21.

16 Aubry-Rozier B, Gonzalez-Rodriguez E, Stoll D, Lamy O. Severe spontaneous vertebral fractures after denosumab discontinuation: three case reports. Osteoporos Int. 2016 May;27(5):1923-5.

17 Polyzos SA, Terpos E. Clinical vertebral fractures following denosumab discontinuation. Endocrine. 2016 Oct; 54(1):271-2.

18 Bone HG, Bolognese MA, Yuen CK, Kendler DL, Miller PD, Yang YC, et al. Effects of denosumab treatment and discontinuation on bone mineral density and bone turnover markers in postmenopausal women with low bone mass. J Clin Endocrinol Metab. 2011 Apr;96(4):972-80.

19 Team RC. A language and environment for statistical computing. Vienna, Austria: R Foundation for Statistical Computing; 2017.

20 Wickham H. ggplot2 Elegant Graphics for Data Analysis Introduction, 2009.

21 Brown JP, Prince RL, Deal C, Recker RR, Kiel DP, de Gregorio LH, et al. Comparison of the effect of denosumab and alendronate on BMD and biochemical markers of bone turnover in postmenopausal women with low bone mass: a randomized, blinded, phase 3 trial. J Bone Miner Res. 2009 Jan;24(1):153-61.

22 Beaudoin C, Jean S, Bessette L, Ste-Marie LG, Moore L, Brown JP. Denosumab compared to other treatments to prevent or treat osteoporosis in individuals at risk of fracture: a systematic review and meta-analysis. Osteoporos Int. 2016 Sep;27(9):2835-44.

23 Anastasilakis AD, Polyzos SA, Makras P. THERAPY OF ENDOCRINE DISEASE: denosumab vs bisphosphonates for the treatment of postmenopausal osteoporosis. Eur J Endocrinol. 2018 Jul;179(1):R31-45.

24 Tsourdi E, Langdahl B, Cohen-Solal M, Aubry-Rozier B, Eriksen EF, Guañabens N, et al. Discontinuation of Denosumab therapy for osteoporosis: A systematic review and position statement by ECTS. Bone. 2017 Dec; 105:11-7.

25 Anastasilakis AD, Yavropoulou MP, Makras P, Sakellariou GT, Papadopoulou F, Gerou S, et al. Increased osteoclastogenesis in patients with vertebral fractures following discontinuation of denosumab treatment. Eur J Endocrinol. 2017 Jun;176(6):677-83.

26 Russell RG. Bisphosphonates: from bench to bedside. Ann N Y Acad Sci. 2006 Apr;1068(1):367-401.

27 Leder BZ, Tsai JN, Jiang LA, Lee H. Importance of prompt antiresorptive therapy in postmenopausal women discontinuing teriparatide or denosumab: The Denosumab and Teriparatide Follow-up study (DATA-Followup). Bone. 2017 May;98:54-8.

28 Reid IR, Horne AM, Mihov B, Gamble GD. Bone loss after denosumab: only partial protection with zoledronate. Calcif Tissue Int. 2017 Oct;101(4):371-4.

29 Uebelhart B, Rizzoli R, Ferrari SL. Retrospective evaluation of serum CTX levels after denosumab discontinuation in patients with or without prior exposure to bisphosphonates. Osteoporos Int. 2017 Sep;28(9):2701-5.

30 Freemantle N, Satram-Hoang S, Tang ET, Kaur P, Macarios D, Siddhanti S, et al.; DAPS Investigators. Final results of the DAPS (Denosumab Adherence Preference Satisfaction) study: a 24-month, randomized, crossover comparison with alendronate in postmenopausal women. Osteoporos Int. 2012 Jan;23(1):317-26.

31 Horne AM, Mihov B, Reid IR. Bone loss after romosozumab/denosumab: effects of bisphosphonates. Calcif Tissue Int. 2018 Jul;103(1):55-61.

32 Marques ID, Araújo MJ, Graciolli FG, Dos Reis LM, Pereira RM, Alvarenga JC, et al. A randomized trial of zoledronic acid to prevent bone loss in the first year after kidney transplantation. J Am Soc Nephrol. 2019 Feb; $30(2): 355-65$. 